\section{THE DIFFERENTIAL DIAGNOSIS OF SCROTAL TUMORS."}

\section{I3Y D. A. K. STEELE, M.D.,}

JROFESSOR OF ORTHCHADIC SURCIERY IN THE COI.HCIV OF MUYSICIAN ANI) SURGEONS OF CHICAGO; SURGKON TO COOK COUNTY HOSIITAL, WTC.

The differential diagnosis of scrotal tumors is not always an casy matter. 'The peculiar anatomical conformation of the parts, the varied morbid alterations of structures normally located, and the frequent intrusion within the scrotal pouch of dislocated or adventitious tissue renders a positive diagnosis difficult, if not indeed impossible in certain cases. It has been our fortune to witness a number of grave errors of judgment as to the nature of tumors within the scrotum, and our misfortune to occasionally participate in these mistakes. When we consider that a scrotal tumor may be a hermia, hyclrocele, orchitis, epididymitis, varicocele, cancer, sarcoma, hematocele, or a complication of two or more of these affections, the reason for an occasional blunder is more apparent-and an allusion to previous mistakes may be a reminder of certain diagnostic points that will serve to warn the members of this Society from pitfalls where others have stumbled.

" $\Lambda$ tumor is a morbid swelling, prominence or growth on any part of the body." Surround this definition with the scrotal walls and you embrace our subject. The scrotum is a pouch formed of the common integuments for the reception of the testicles. It is evenly divided externally into two halves by a prominent ridge, a continuation of the raphe from the under part of the penis to the perineum. The skin of the scrotum is thin like that of the eyelicls, and darker in color than most other parts. It is very loose and extensible, as evidenced in connection with scrotal hernia or very large hydroceles.

The dartos is a thin layer of contractile tissue of a reddish-grey color immediately beneath the skin, and intimately connected with its inner surface. Bow. man considers the dartos to be muscular and composed of "unstriped elementary fibres." Scrotal movements by the dartos are involuntary and peristaltic or vermicular in character. Contraction is excited by cold, fright or venereal orgasm; relaxation by heat, old age, debility. Galvanism produces no effect. Beneath the dartos there is a quantity of loose cellular tissue. Underlying this we find the superficial spermatic fascia continuous with that cov. ering the lower part of the abdominal walls, and descending to form a sheath for the spermatic cord and enveloping the testis.

Beneath this is the cremaster muscle, which is interesting from a diagnostic point of view on account of its action in elevating the testicles to the abdominal rings, and the fact that sometimes this power of retraction is entirely volitional and might be used by a malingerer to simulate an inguinal hernia. Pre supposing a thorough knowledge of the anatomy of the testis on the part of my auditors, a detailed description is not needed; it will only be necessary for us to remember the relation of the parts composing these glands. First, the tunica vaginalis,

3 Read before the Chicago Medical Society, July 19,1886 a delicate serous membrane in the form of a shut sac, enveloping by means of its outer layer all of the testis except its posterior edge and lower extremity, is derived from the peritoneum. The line of attachment is about five lines from the inferior extremity of the testis. Second, the tunica albuginea, a dense fibrous layer forming the covering proper of the gland, but not including the epididymis. Third, the gland structure proper, consisting of the seminiferons tubes, blood-vessels, lymphatics and nerves. Fourth, The epididymis is a continuation of the gland proper, containing the excretory ducts which empty into the vas deferens, which is the real excretory duct of the testicle.

$\Lambda t$ an early period of foctal life the testes lie behind the peritoneum in the region of the kidneys, being loosely attached to the peritoneum by the mesorchium. The inguinal canal has already been formed, and is occupied by a cord.like structure, the $g^{\text {ruther }}$ naculum testis, which extends from the bottom of the scrotum to the testis, to the under surface of which it is attached, and as the testicle gradually descends during the last months of foutal life the gubernaculum testis shortens and guides the way. At the same time the peritoneum enters the inguinal canal in advance of the testicle, descending in a loose fold as the gubernaculum contracts and draws the testicle down after it. The descent of the testicle is usually completed shortly before the birth of the fortus; and, as a rule, the neck of the peritoneal sac is obliterated by occlusion before the child is born, thus cutting off all communication between the tunica vaginalis and the peritoneal cavity. This occlusion may be incomplete, thus giving rise to congenital hydrocele, or it may occur at two or three different points, leaving free serous spaces intervening, occasioning encysted hydrocelc-or hydrocele of the cord. So much, then, for an outline of the anatomical points of our subject.

For diagnostic or clinical purposes tumors of the scrotum may be divided into two general classes, viz. : reducible and irreducible-reducible tumors including all hernic (except strangulated), varicocele, and congenital hydroceles. Irreducible tumors of the scrotum would include all tumors connected with the testicle, all hydroceles (except congenital), and strangulated hernia. Tumors of the tegumentary surface of the scrotum, such as inflammatory odema, elephantiasis and epithelioma, are usually so characteristic in their history as to offer no special impediments to a correct diagnosis.

Scrotal hernia may be mistaken for:

I. Hydrocele of tunica vaginalis, cord, or encysted hydrocele.

2. Sarcocele of the testicle, simple, tuberculous, cystic or malignant.

3. Varicocele.

4. Hamatocele.

5. Bubo.

6. Undescended testis.

In scrotal hernia, as a rule, the tumor is soft and doughy to the touch, light weight, smooth and regular, painless unless inflamed or strangulated, of sudden advent from above downwards, resonant on percussion, fills the inguinal canal, has cough impulse, 
gurgles, is of normal color, opaque, may exist on either side, the spermatic cord is concealed, does not fluctuate, aspiration is negative, bowels may be embarrassed. It can be reduced unless the hernia is strangulated or incarcerated.

I. (a) In hydrocele of the testicle the tumor is ovoid or pyriform, develops slowly from below upwards, is firm, tense and elastic, fluctuates, is translucent, dull on percussion; is irreducible; spermatic cord is neither concealed or displaced; the inguinal canal is empty; bowels unaffected; aspiration reveals fluid.

(b) In hydrocele of the cord the difference is that the tumor is circumscribed, encysted, and sometimes reducible, situated usually near the epididymis, but returns after reduction irrespective of position.

(c) In congenital hydrocele the fluid clisappears completely within the peritoneal cavity by compression of the tumor for a short time.

2. (a) In sarcocele of the testicle the tumor is usually hard and resistant, heavy, often nodular and irregular; painful; grows slowly; dull or flat on percussion. The inguinal canal is empty; no impulse on coughing; bowels unaffected; irreducible; no auscultatory sounds. Simple sarcocele is chronic orchitis. Both the epididymis and body of the gland are affected. The cord is usually thickened. Ab. scess of the organ may occur. It is caused usually by an injury followed by inflammatory deposits.

(b) Tubercular sarcocele is met with most frequently in early manhood, and may occur in any constitution; in the strong and robust as well as the weak and cachectic; and although often associated with tuberculerization of other organs, it is common enough to find the tuberculous nidus in the epididy. mis, not as a sequence of gonorrhoeal inflammation or some slight injury followed by inflammatory infiltration-as was formerly believed-but as a coincident.' 'The progress is slow and insidious. The gland at first moderately enlarges with little or no pain, the hypertrophy being especially marked in the globus major. Presently the outline of the tumor becomes craggy or nodulated, and circles around the testicle from behind forwards in the form of a crescent. After several months, this adventitious tissue exceeds in size the testicle proper, and then it begins to soften at points and one or more abscesses burst and discharge a thin shreddy pus. 'The vas deferens is greatly enlarged.

(c) In syphilitic sarcocele or gummata the history of the patient guides us in the diagnosis. Also, we find that the body of the gland is usually the seat of the infiltration which takes place in the connective tissue between the tubuli seminiferi, the epididymis undergoing little if any enlargement. The cord and vas deferens are unaffected. There is little or no tenderness, and the peculiar sensation elicited by squeezing a healthy testicle is absent. The tunica albuginea is very greatly thickened. Hyclocele is

1 The bacilli tuberculosis which were formerly prolably disporting in the blood in a state of isnocuous destsetude, now finding a fertile soil in the increased vascularization of the part, proceed to inhabit, multiply and take possession of the part to its ultimate destruction--just as other microbes have an affinity for the epiphysis of long bones where the bloud current is sluggish. a frequent complication and tapping is often required to establish a diagnosis.

Cystic tumors of the testis closely resemble hydrocele, and differ chiefly in being opaque instead of translucent. Aspiration should be practiced before pronouncing positively upon their character.

Cancer of the testicle primarily invades the body of the gland, and almost invariably assumes the encephaloid form. Most observers doubt the existence of other varieties of malignant disease in this organ. The development of the disease is rapid. The patient has a sensation of weight, pain and dragging in the testis, the scrotum becomes distended, reddish or purplish, and the superficial veins are seen to be enlarged. The skin adheres to the gland, ulceration occurs, fungus protrudes, the inguinal glands are secondarily involved, and the patient by this time presents the characteristic cancerous cachexy.

3. In varicocele the tumor is knotty and irregular like a bag of worms, bluish in color; most frequent upon the left side; increases in size upon the application of heat; develops gradually; is dull on percussion; fuctuation doubtful; spermatic cord not affected; inguinal canal not involved. No cough impulse. It reduces spontaneously by any position that favors increased venous return, but returns immediately when the patient stands up notwithstanding pressure at the ring. There is weight and dragging in the scrotum.

4. In hamatocele the advent is sudden; usually traumatic in origin; grows from below up if spontaneous; fluctuates until coagulation occurs; is at first soft, and hard after coagula form. It is pyriform in shape ; ecchymotic; irreducible; heavy; dull on percussion. The spermatic cord is unaffected, and the inguinal canal empty. There is often pallor and prostration from loss of blood. The bowels are unaffected.

5. Bubo is seldom mistaken for a scrotal tumor, and it is unnecessary to name differential points.

6. An undescended testicle is painful, and pressure upon it yields a peculiar sickening sensation. It is found wanting upon the side occupied by the tumor, and the scrotum is imperfectly developed upon the same side. It is sometimes mistaken for a bubonocele.

In order that it may not be thought that the somewhat minute enumeration of diagnostic points mentioned in connection with these various scrotal tumors is tedious or unnecessary, I beg to present the following cases which have fallen under my observation during the past few years. In all of them a more or less serious error of judgment occurred.

Case 1.-S. J. J., ext. 3o. On Sept. 16, I883, Mr. $\mathrm{J}$. called upon me, stating that he was suffering from a hydrocele and wished a permanent cure; that he had consulted Dr. Roswell Park, who advised tapping it, but that as 1)r. Park was about leaving the city, his family physician, Dr. Doering, advised him to place hinsself under my care for a radical cure. Mr. J. stated that when he was a boy while in swimming one day his attention was directed to the fact that the right side of the scrotum was larger and heavier than the left, but that he had never paid any attention to it until about a year previous to the time he 
called upon me, since which time he had noticed a gradual enlargement that caused no pain or discomfort except a sense of weight and a tired feeling in that side; but as it was steadily and slowly increasing in size he decided to have it removed. It was soft and fluctuant to the touch, pyriform in shape, slightly translucent; inguinal canal full and prominent, and from the history and physical appearance I concluded that it was a hydrocele complicated with a tumor connected with the testicle, and, having some doubt as to the exact nature of the growth, I took him to Dr. E. Andrews, who concurred in the opinion of a hydrocele and thought the tumor was possibly a sarcoma or cystic testicle, and advised emptying the tunica vaginalis and then examining the tumor more satisfactorily.

On Sept. 22 I aspirated the tunic in the presence and with the assistance of Dr. Roler, obtaining about two ounces of clear serum instead of a pint or more that $I$ expected. I injected sac with 5 per cent solution of carbolic acid, and kept him in bed for a few days. I then concluded that the testicle was cystic and advised its removal, which was readily agreed to.

On Oct. 5 , in the presence of Drs. Andrews, Doering, Beery, Stowell, and two or three students, I operated by making a long free incision down through the successive layers upon the tunica vaginalis, which I then loosened and raised up to examine more carefully. It was soft, fluctuant and opaque. I made a free incision into it, and instead of fluid out gushed a mass of omentum that had distended it, closely surrounding and masking the testicle. A small quantity of serum was present, an ounce or two perhaps. All doubt now disappeared and we had to deal with a case of incarcerated congenital omental hernia of the tunica vaginalis-the testicle was perfectly normal. The omental mass was tied in sections with catgut and cut off short, being left as a plug in the inguinal canal. All bleeding points were ligated with catgut. The testicle was returned within the tunic, a drainage-tube inserted, and the wound washed out with a carbolized solution and antiseptic dressing applied.

Mr. J. made a steady and uninterrupted recovery, resuming work in less than two months. 'The only complication was a circumscribed cellulitis extending along the cord, that appeared about two weeks after the operation, and was treated by hot poultices and blisters for some ten days, when the induration disappeared. I have seen the same complication in three other cases of operations upon the testicle, and believe it is a common occurrence. In this case the error was made of not attaching sufficient importance to the filling up of the inguinal canal.

Case 2,-S., xt. 29, a healthy young man, applied to me in August, $188 \mathrm{I}$, for relief from a subacute epididymitis that came on a few weeks before as the result of a slight injury. It was treated by a suspensory, strapping and alterative ointments, but steadily and slowly enlarged until October, when it became nodular and fluctuant at two points, encroaching more and more upon and around the testicle. Dr. Gunn saw him about this time and opened an abscess, regarding it as a simple abscess of the connective tissue and not involving the gland. It continued to discharge, the testicle increasing in size and the health of the patient declining until December, when a consultation was held. Drs. Gunn and Fenger advocated the removal of the diseased organ. Dr. Andrews favored temporizing. It was removed on January 11,1882 , and found to be tubercular. This operation was also followed by a circumscribed peritonitis and cellular induration along the cord. Prompt recovery followed, and he has enjoyed excellent health since.

Case 3.-Boy, at. 8 years. I was called to reduce a strangulated hernia, when I found a simple hydrocele.

Case 4.-W., at. 6o. Had been troubled with an old inguinal hernia for years. On November 10, I880, it came down and could not be returned. Four days later an operation was made, when, on opening the peritoneal sac, a cyst was found that had acted as a valve at the neck of the canal, preventing the return of the bowel by taxis. This condition was not suspected prior to the operation.

Case 5.-Chas. M., at. 23. Had a right oblique inguinal hernia for ten years, supposed to have come from the kick of a horse. On February 6, 1883 , it became strangulated. At the examination a large painful tumor was found. Within the external ring in the inguinal canal a smaller tumor was detected, which proved to be an undescended testicle. The gut was returned to the abdominal cavity, and as the testicle was useless and in the way of wearing an appropriate truss, it was removed. The young man made a good recovery.

Case 6.-J. W., at. 46, had a congenital left inguinal hernia. On September I5, 1882, the bowel came down and became strangulated. Thirteen days later an operation was made, and a large mass of omentum and a knuckle of ileum found. 'These were returned, when the scrotum was found to be occupied by an immense hypertrophy of the cord and epididy. mis measuring eighteen inches in length and weighing twelve ounces. This was cut off and the stump tied at the ring. 'The patient died from exhaustion. This case is reported by Mr. John $\mathrm{I}$. Atlee, and is certainly a rare complication.

Case 7.--In December, 1883 , Ir. John Bell, of Benton Harbor, Mich., brought me a farmer, in consultation, who had multiple cysts of the cord. He had been treated by a quack for spermatorhoa, said quack telling him that there were 478 cords running from his testicles to his heart, and if he had any operation done and a single one of those cords was wounded, instant death would ensue. I aspirated the cysts, and they have not refilled as yet.

Case 8.-Tuberculosis of testicle mistaken for cancer.

In conclusion I only desire to say that the diagnosis of scrotal tumors requires careful study and the employment of all the means at our command. No symptom should be lightly passed over and we should accept nothing on trust, but verify all statements ourselves. Diagnosis should be by exclusion.

I80x State Street, Chicago, Ill. 